\title{
Panel discussion section $\mathbf{J}$
}

\author{
Chair: Gregg A. Wade
}

Section organizer \& KeY-note SPEaker: Stefano Bagnulo

InVITED SPEAKERS: N. Piskunov, P. Padovani

\section{Discussion}

WADE: For Padovani: Is it straigthforward to implement our own catalogs into the Virtual Observatory (VO)?

PADOVANI: Very easy. There is a button to load your own data, and that includes a list of sources. The table has to contain one record per line, each field separated by a TAB. Once loaded, your own catalog can be overlayed on any image and cross-correlated with any catalog in the public domain.

ŠKODA: How do you handle in the VO the ambiguity of positions when cross-correlating several catalogues (mainly faint objects)? Can you add more information to search?

PADOVAnI: Certainly. The present tool is very simple but we are planning to improve it and add more features, including a probabilistic determination of cross matches.

MathYS: How do you retrieve moving targets from AVO?

PADOVANI: This is an issue which is still open. The time domain is still largely unexplored but it will certainly be considered.

Adelman: Elizabeth Griffin has pleaded the case for getting spectroscopic plates scanned, which I think is quite important. An equally important case can be made for photometric measurements using photomultiplier tubes and CCDs, most of which have never made their way into the literature except perhaps graphically. This is especially the case prior to journals accepting electronic tables.

PADOVAnI: The usual answer is the following: the VO cannot do that. It is up to the data providers or archive centres to do that. We do not have the manpower. We can tell you what to do and how to do it, but we cannot do it for you, unfortunately.

AdELman: Still someone or some organization needs to encourage people with this type of data to submit it or astronomy soon loose much of the photometry obtained in the past 50 years.

LANDSTREET: It would be very useful if the VO could provide tools for converting typed pages (old tables, etc.) to ASCII files. For deciding how to consolidate old data in the hands of individuals, it would be good to involve IAU commissions such as variable stars, photometry, polarimetry, etc. 
PAdovani: Commercial software exists which does Optical Character Recognition (OCR) and converts any printed material to ASCII files. I am not sure it is the VO's role to provide anything which goes beyond that. As I have just said, it is up to the data providers or archive centres to do that.

BAGnulo: It seems to me that the A-star community is very interested in the research possibilities offered by the VO. Paolo Padovani has also presented a few practical possible applications. Another perspective of the future astronomical research is the use of Extremely to Overwhelmingly Large Telescopes, and I have a question for the all of us present in this room. If you get observing time for the A-star research with a 100-m telescope, what would you do?

Piskunov: To use a 100-m telescope for a single object project will be much easier because it is much easier to correct the small field of view for atmospheric disturbances and obtain a nearly diffraction limited image on the optical axis of the telescope which means that high-resolution spectroscopy is the most logical thing to do.

BAGNulo: The question is high-resolution spectroscopy of what? Why do you need a 100-m telescope to do the high-resolution spectroscopy of A-stars? What would you do that cannot be done with smaller telescopes? To this question, some time ago John Landstreet answered that we should study whether the A-star phenomenon is just a local phenomenon or it is common in other galaxies. This we can do it only with extremely large telescopes.

WADE: Can we resolve individual A-type star in nearby galaxies?

WeIss: It has been done. There has been a discovery of CP stars by Maitzen and coworkers.

BAgnulo: Still in the Large Magellan Cloud, that is close by our galaxy. In any case they have been identified only via $\Delta a$ photometry. We do not have spectroscopic observations and thus do not know the detailed chemical characteristics of these stars.

LANDSTREET: I would like to make two comments. First: we may hope to get highresolution spectrograms on new large telescopes because the planet-finding community may want them. Second: a lot of useful information is available for clusters. But to study OLD stars in OLD clusters, which are rare, we need to observe to great distances. Furthemore, we can observe stars (Am, Ap, HgMn, etc.) formed in really different environments from our local one by looking at the nearby dwarf galaxies.

PAUnzen: We do not even know the incidence of $\mathrm{CP}$ stars for open clusters within two kpc around the Sun. Why go for dwarf galaxies? By contrast, there is a need for classification resolution spectroscopy in our Milky way. Especially for field stars from $10<V<15 \mathrm{mag}$.

Corbally: Paunzen's remarks prompt me to point out that the power of the "Nearby Stars" project (see C. J. Corbally \& R. O. Gray, 2005, These Proceedings, IP1), which I am doing with Richard Gray, Bob Garrison, and others, is in its homegeneous approach to a VOLUME limited sample (A0pe). The latter point is thanks to Hipparcos data. In the future we can look to such satellites as GAIA to let people confidently survey larger volumes, while more automated techniques will help them cope with the numbers of 
object that are cubed. I am sure they will find interesting science, perhaps concentrating on particular parts of the HR diagram such as A-stars. It is very worthwhile, as well as fun, to get to know one's neighbours.

WeISS: I would like to come back to the question raised by Bagnulo To me it appears a little bit as a virtual problem. At first I was quite sure that we will find a lots of excellent scientific reasons why we would like to use a 100-m telescope. But then I see the hurdle of the telescope time allocation committee. Already it is nearly impossible to convince a reasonable scientist that it is necessary to point the $8-\mathrm{m}$ telescope to a star which you can see with the naked eye. I am having troubles with 8-m telescopes and the naked eye visibility of a star and transferring them to a 100-m telescope. Then I see there is no chance to get time unless you promise to observe an Ap star in a quite remote galaxy...

BAgnulo: I do not think it is a too serious problem. In fact, a lot of stellar science is being done at the VLT, and a large number of CP stars have been already observed with UVES. Don Kurtz has presented at this conference the preliminary results of a lot observations of roAp stars obtained also with UVES. However it is true that the largest telescopes are designed mainly to do extragalactic and cosmological studies, or to search for life on other planets, so we should be ready to prepare an adeguate scientific case for the studies of A-stars with extremely large telescopes.

MATHYS: It shoud be stressed that a very significant factor of the time at ESO's VLT is devoted to observations of stars of the Bright Star Catalogue. On some of the instruments, there is actually more bright time than dark time allocated. I can only urge again the members of this comunity to get rid of the misconception that stellar astronomers have little access to $8-10 \mathrm{~m}$ telescopes, and to encourage them to submit observing time applications with the best possible science cases.

PRZYBILlA: When talking about science to be done with a $100 \mathrm{~m}$ telescope one has to remark that it will most efficiently operate in the IR. This will allow to study objects in otherwise inaccessible environments, like, e.g., A-stars in the Galactic centre. To do so, we should start to prepare for this now, by extending the observed wavelengths range and gain experience in the IR.

WADE: Of the existing $8+\mathrm{m}$ telescopes, the Keck, the largest telescope in the world, what has it done which is remarkably relevant to A-star research? The only such telescope we have heard about is VLT!

Piskunov: The Keck time allocation procedure makes it difficult to get time for A-star research. At Gemini is possible, but there is very limited high-resolution capabilities.

PRZYBILlA: Spectroscopy with Keck on A-stars is in fact being done. The group around Kudritzki et al. has already started multi-object spectroscopy of A-supergiants in galaxies of the local group, and publications on that are pending.

Mathys: The use of $8-10 \mathrm{~m}$ telescopes by the A-star community seems to reflect the difference of population of astronomical community between North America and Europe. This to me appears perfectly illustrated by the composition of the assembly in this room.

Adelman: I also want to make a few comments about small telescopes. Small telescopes especially those which are automated at good sites, and properly equipped can gather 
a substantial amount of photometry. Those with photomultiplier photometry could be upgraded to CCD photometry. The forthcoming ASTRA spectrophotometer will be able to measure energy distributions of some 40000 bright (-1 to 9 mag) stars per year.

STÜTZ: For Bagnulo: Combining interferometry and spectroscopy at the VLT has been brought up some time ago. When, do you think, that possibility will be working and what will be the chances that one will get observing time for such a setup doing A-star research?

BAGNulo: I am not the right person to answer, as I just heard about a proposal to combine UVES with VLTI. This project is done within the European Interferometry Initiative (under OPTICON) and shall be one of those to be proposed as second generation VLTI-instruments. A paper has been presented by Quirrenbach in the last SPIE meeting in Glasgow. 